\title{
Factores de riesgo de mortalidad perinatal en hospitales de la seguridad social peruana: análisis de los datos del Sistema de Vigilancia Perinatal de EsSalud
}

\author{
Risk factors for perinatal mortality in Peruvian social security hospitals: EsSalud's Perinatal \\ Surveillance System data analysis
}

\author{
Alejandro Arrieta-Herrera ${ }^{1}$, Gloria Riesco de la Vega ${ }^{2}$ \\ ${ }^{1}$ Indiana University School of Public and Environmental Affairs. Indianapolis, Indiana, EE UU. \\ ${ }^{2}$ Programa Nacional Materno - Perinatal, EsSalud. Lima, Perú.
}

\begin{abstract}
Resumen
Introducción: El estudio utiliza la información del Sistema de Vigilancia Perinatal (SVP) de la red hospitalaria del Seguro Social de Salud del Perú (EsSalud) para identificar factores de riesgo que inciden en la mortalidad perinatal. EI SVP incluye policlínicos y hospitales en todo el Perú, con diferentes niveles de capacidad resolutiva. El estudio agrupa hospitales según mayor y menor capacidad de resolución, para investigar la magnitud de la incidencia de los factores de riesgo en cada grupo. Finalmente, el estudio resalta la importancia de mantener un sistema de información perinatal eficiente y ágil que pueda ser integrado entre diferentes redes de salud en el país, con el objetivo de reducir la mortalidad perinatal nacional. Objetivos: El primer objetivo fue encontrar los principales factores de riesgo de mortalidad perinatal en la red hospitalaria de EsSalud. El segundo objetivo fue identificar diferencias en la magnitud de la incidencia de dichos factores en hospitales de menor y mayor capacidad resolutiva. Diseño: Estudio descriptivo, comparativo y transversal. Lugar: Red nacional de hospitales de EsSalud, Perú. Participantes: Madres y sus recién nacidos. Intervenciones: Se utilizó información clínica del SVP de EsSalud para estimar probabilidad de muerte perinatal, basado en un modelo logístico multivariado, que incluyó 108813 nacimientos correspondientes a madres y sus recién nacidos en EsSalud, en los años 2005 y 2006 . Principales medidas de resultados: Muertes perinatales. Resultados: La anomalía congénita fue el principal factor de riesgo de la mortalidad perinatal en los hospitales de menor y mayor capacidad resolutiva ( $O R=30,99$ y 15,26, respectivamente), seguido por prematuridad menor a 32 semanas $(O R=15,68$ y 4,20$)$ y peso mayor a 4000 gramos $(O R=4,17$ y 3,87$)$. Factores de riesgo de la madre también resultaron asociados a mortalidad perinatal, pero en menor magnitud, resaltando el sangrado genital después de la semana 24 del embarazo $(0 R=4,23$ y 3,81) y otras entidades obstétricas diferentes a anemia, preeclampsia y rotura prematura de membranas $(O R=4,53$ y 1,76$)$. Conclusiones: Los factores de riesgo identificados son consistentes con la literatura sobre mortalidad perinatal. En los hospitales con mayor capacidad resolutiva, la incidencia de los factores de riesgo sobre mortalidad es menor, a pesar de que estos concentran pacientes con riesgo obstétrico alto y por tanto mayor mortalidad. Los hospitales de menor capacidad resolutiva requieren focalizar recursos más adecuadamente y mejorar sus sistemas de referencias. EI SVP de EsSalud resulta una herramienta válida, consistente y útil para un mejor monitoreo y control de la mortalidad perinatal.
\end{abstract}

Palabras clave: Mortalidad perinatal; hospitales; factores de riesgo; salud infantil, Perú.

\begin{abstract}
Introduction: This study uses information from the Perinatal Surveillance System (Sistema de Vigilancia Perinatal, SVP) of the Peruvian social security hospitals (EsSalud) to identify risk factors for perinatal mortality. The SVP includes health centers in all the country with different levels of hospital care. The study groups hospitals based on higher or lower levels of care in order to quantify the incidence of risk factors in each group. Finally, the study highlights the importance to keep an efficient clinical database that could be integrated with other health organizations in the country, in order to joint efforts to reduce perinatal mortality in Peru. Objectives: The first goal was to find the main risk factors for perinatal mortality in EsSalud's hospitals. The second goal was to identify differences in the incidence of these factors on hospitals with higher and lower levels of care. Design: Descriptive, comparative and transversal study. Setting: EsSalud national hospitals net, Peru. Participants: Mothers and their newborns. Interventions: Clinical data from the SVP was used to estimate probability of death during the perinatal period; 108813 newborns and their mothers whose deliveries were in years 2005 and 2006 were studied. Estimations were based on multivariate logistic models. Main outcome measures: Perinatal mortality. Results: Congenital anomaly was the most important risk factor for perinatal mortality in hospitals of lower and higher level of care $(\mathrm{OR}=30,99$ and 15,26 respectively), followed by prematurity below 32 weeks ( $O R=15,68$ and 4,20) and weight above 4000 grams $(\mathrm{OR}=4,17$ and 3,87$)$. Maternal risk factors were also associated with perinatal mortality but with a lower incidence. The most important were genital bleeding after 24 weeks of pregnancy $(O R=4,23$ and 3,81), and other obstetric entities different from anemia, preeclampsia, and premature membrane rupture $(0 R=4,53$ and 1,76). Conclusions: Risk factors identified in this study are consistent with medical literature on perinatal mortality. In hospitals with higher level of care the incidence of risk factors on mortality is lower, even though they concentrate the most risky patients and consequently the highest mortality. Hospitals with lower level of care require to focus resources on main risk factors, and to improve their referral systems. EsSalud's SVP is a valid, consistent and useful tool to monitor and control perinatal mortality.
\end{abstract}

Key words: Perinatal mortality; hospitals; risk factors, child welfare, Peru.

\section{INTRODUCCIÓN}

La reducción de la mortalidad infantil es el cuarto objetivo de desarrollo del milenio de las Naciones Unidas. La meta es que, para el año 2015, la mortalidad de niños menores de 5 años se reduzca en dos tercios respecto a 1990. De acuerdo a un reciente informe de Unicef ${ }^{(1)}$,
América Latina ha reducido la tasa de mortalidad de menores de 5 años en $40 \%$, entre 1990 y 2007, y en particular el Perú la ha reducido en más de $74 \%$ (de 78 a 20 muertes por mil nacidos vivos), reubicándonos por debajo del promedio latinoamericano y alcanzando uno de los objetivos más importantes de salud pública del país ${ }^{(2,3)}$. Los mayores logros en esta reducción han sido en muertes durante el periodo posneonatal, es decir, en el periodo comprendido entre los 29 días y los 5 años de edad.

Sin embargo, dentro de la mortalidad infantil, la población más expuesta es la de neonatos con menos de una semana de vida (periodo neonatal temprano). 
De acuerdo a información mundial de la $\mathrm{OMS}^{(4)}$, cerca de un tercio de muertes en niños menores de 5 años se produce en la primera semana de vida. En el año 2004 esto representó 2,8 millones de muertes. La cifra es más alarmante si se incluye los partos con productos natimuertos (muertes fetales ocurridas durante el periodo posterior a las 22 semanas de gestación hasta el parto), que en el mismo año sumaron 3,1 millones de muertes. Eso ha motivado que los esfuerzos internacionales en reducir la mortalidad infantil se concentren ahora en la mortalidad perinatal (muertes ocurridas en fetos con más de 500 gramos o más de 22 semanas de gestación, más muertes durante los 7 días después del nacimiento -periodo neonatal temprano), la cual constituye un indicador de los riesgos ligados a la reproducción y a la atención prenatal, durante el parto y posparto. Según datos de la OMS para el 2004, la mortalidad perinatal alcanzó la tasa de 43 por mil nacimientos a nivel mundial y 17 por mil en América del Sur. En el Perú, la tasa se estimó en 14 muertes perinatales por cada mil nacimientos, ubicando al país en mejor posición que el promedio Latinoamericano.

La estimación de mortalidad perinatal de la OMS se basa en encuestas nacionales, como la encuesta demográfica y de salud (DHS o ENDES), que se aplica sistemáticamente a diversos países en desarrollo. Sin embargo, existen importantes limitaciones en la estimación de tasas de mortalidad perinatal cuando se utiliza encuestas nacionales de salud, sobre todo en el componente de natimuertos, que usualmente es subestimado por los encuestados. Cuando el objetivo es entender los factores de riesgo que explican la mortalidad perinatal, estas limitaciones generan sesgos de estimación mayores, reduciendo la posibilidad de utilizar estos resultados para establecer políticas de salud más efectivas. Una mejor alternativa de medición y estudio de la mortalidad perinatal es a través de datos administrativos hospitalarios. Lamentablemente, los datos administrativos en países en desarrollo son precarios y poco confiables. Por ejemplo, el Sistema Informático Perinatal (SIP) administrado por el Centro Latinoamericano de Perinatología y De- sarrollo Humano (CLAPDH) considera fichas con excesivos requerimientos de información que repercuten en errores de reporte, registro incompleto de fichas e incluso inaplicabilidad del SIP ${ }^{(5,6)}$. En el caso del Perú, los autores encontramos por ejemplo que la ficha puede tardar 20 minutos en ser llenada adecuadamente y que, en muchos establecimientos, los nacimientos registrados en el SIP no llegan al 20\% del total de nacimientos reales, experiencia que ha sido reconocida por el CLAPDH ${ }^{(7)}$.

Pese a los problemas del SIP, algunos estudios han podido utilizar esta información para un grupo limitado de establecimientos ${ }^{(8,9)}$, usualmente ubicados en ciudades urbanas de densidad poblacional alta. Sin embargo, un mayor entendimiento de la mortalidad perinatal requiere acceso a información a nivel nacional. En esa línea, el presente estudio utiliza datos clínicos del Sistema de Vigilancia Perinatal (SVP) implementado hace 10 años sobre una amplia red de hospitales de la seguridad social peruana (EsSalud), ubicados en diferentes zonas geográficas del país. EsSalud es el segundo proveedor de servicios de salud en el Perú. Más de 20\% de partos en el país son atendidos en la red hospitalaria de la seguridad social, siendo el Ministerio de Salud (Minsa) el principal proveedor, con cerca de $40 \%$ de atenciones.

El primer objetivo de este estudio fue determinar los principales factores de riesgo que explican la mortalidad perinatal en la red hospitalaria de EsSalud. El estudio se concentró en dos grupos de establecimientos de salud, diferenciados por la capacidad resolutiva en la atención materno-perinatal dentro de la red hospitalaria. El primer grupo comprendió hospitales con capacidad resolutiva alta, los cuales cuentan con mayores recursos humanos y tecnológicos para atención de partos de mayor riesgo. Las políticas de transferencia hospitalarias hacen que estos establecimientos atiendan los casos de más riesgo. Por otro lado, el segundo grupo de establecimientos con menor capacidad de recursos atiende mayoritariamente partos sin problemas. El segundo objetivo de este estudio fue cuantificar la incidencia de factores de riesgo sobre la mortalidad perinatal en cada uno de estos dos grupos de hospitales.

\section{MÉTODOS}

Se utilizó información de nacimientos del SVP de EsSalud correspondiente a los años 2005 y 2006, así como información agregada del año 2000, con propósitos comparativos. El SVP fue creado en 1997, gracias a un convenio institucional con el gobierno de Cuba. Fue desarrollado conjuntamente con un grupo de médicos cubanos como una herramienta informática del Programa Nacional de Perinatología. El objetivo fue contar con información confiable y en tiempo real de la salud materna y neonatal, la cual permite realizar una gestión basada en evidencias y un mejoramiento continuo de la atención perinatal.

Cabe señalar que el sistema hospitalario de EsSalud está definido sobre 27 redes asistenciales a nivel nacional, en tres niveles de atención. El primer nivel de atención incluye policlínicos, centros y postas médicas destinadas a brindar atención primaria de salud (prevención, promoción y recuperación de capa simple); el segundo nivel es dado en hospitales de niveles I y II, inicia la atención hospitalaria; y, por último, el tercer nivel de atención es dado por los hospitales III, IV, nacionales e institutos, que brindan atención de complejidad alta. Esto determina que haya una mayor concentración de riesgo obstétrico en los hospitales III, IV y nacionales, lo que implica el funcionamiento de un sistema de referencias y contrarreferencias entre los establecimientos de la Red y entre las Redes. La población adscrita al sistema corresponde mayoritariamente al sector de trabajadores formales, lo cual define el perfil epidemiológico de este grupo y lo distingue de la población más pobre sin acceso a salud o con acceso a través de la red de atención del Minsa.

El SVP entró en operación en el año 1998 y empezó a funcionar con una muestra de 27 establecimientos de EsSalud, denominados hospitales centinela. La selección de hospitales centinela se tomó teniendo en cuenta el número de partos y cesáreas atendidos, la ocurrencia de muertes perinatales y su representatividad geográfica, estadística y de nivel de atención dentro de EsSalud. En 1998, los nacimientos en los hospitales centinelas representaron el 70\% del total de nacimientos de la institución y el $83 \%$ 
de la mortalidad perinatal. En el 2005 y 2006, años escogidos para el presente estudio, el SVP se había ya consolidado como una herramienta fundamental para la obtención de indicadores maternos y neonatales institucionales, contando en ese momento con 56 hospitales centinelas, que representaban el $100 \%$ de hospitales nivel IV y III, el 70\% de hospitales II y 30\% de hospitales I, los cuales cubrían el $85 \%$ de los nacimientos y $90 \%$ de las muertes perinatales institucionales. Cabe mencionar que, en el año 2007, se desarrolla la versión en web del sistema, la cual integra a todos los hospitales a nivel nacional. Esta versión estuvo en proceso de implementación hasta el año 2008, razón por la cual no formó parte de este estudio.

En el periodo de estudio, se reportó 56839 nacimientos en todo el año 2005, y 55722 ingresos en todo el año 2006 . Esto correspondió a los 56 hospitales centinelas que formaron parte del SVP durante ese periodo. Para el estudio, se descartó la información de establecimientos con menos de 40 nacimientos anuales ( 7 establecimientos, $0,11 \%$ de la muestra) y aquellos ingresos con registros incompletos (0,88\% de la muestra). Para mantener consistencia con la definición de mortalidad perinatal, se excluyó también los nacimientos con pesos al nacer menores a 500 gramos y periodo gestacional menor a 22 semanas (1 caso). Finalmente, el análisis excluyó los nacimientos múltiples $(2,41 \%$ del total de los nacimientos reportados), debido a que el impacto de varios factores de riesgo es diferente entre partos múltiples y únicos $(10,11)$ y a que la creciente fertilización in vitro podría distorsionar los resultados. La muestra final consideró un total de 108813 nacimientos.

La probabilidad de muerte perinatal se estimó con un modelo Logit multivariable que incluyó como factores de riesgo características de la madre y el recién nacido. En el caso de madres, se incluyó edad en años, dos indicadores para pacientes menores a 19 años y mayores a 35 años, número de partos anteriores, número de abortos, un indicador para reportar alguna enfermedad durante el embarazo (hipertensión arterial, diabetes, asma bronquial, cardiopatía, infección urinaria y otras enfermedades) y 5 indicadores para identificar entidades obstétricas, como anemia, preeclampsia, sangrado genital después de la semana 24 del embarazo, rotura prematura de membranas y otras entidades. En el caso de recién nacidos, se incluyó tres indicadores de edad gestacional (menor a 32 semanas, entre 33 y 36 semanas, y 42 semanas a más), variaciones del peso al nacer por 100 gramos, tres indicadores de peso bajo (menor a 1000 gramos, entre 1001 y 1500 gramos y entre 1501 y 2500 gramos), un indicador de sobrepeso (mayor a 4000 gramos), un indicador por sexo femenino y un indicador de anomalía congénita. En el caso de anomalía congénita, se observó valores incompletos (missing values) cuando el estado al nacer fue comunicado como muerto antes del trabajo de parto $(0,63 \%$ de la muestra final). Para no excluir estas observaciones, los valores incompletos fueron atribuidos usando imputación múltiple, que resulta ser un método eficiente cuando la fracción de valores incompletos es pequeña, como en nuestra muestra ${ }^{(12)}$.

Existen importantes diferencias entre hospitales con distintos niveles de atención. En particular, los establecimientos de mayor capacidad resolutiva (hospitales III, IV y Nacionales) atienden un mayor número de nacimientos (cerca de 60\% de los nacimientos en EsSalud) y absorben los casos de mayor riesgo (tasa de mortalidad perinatal promedio de 16 por mil nacidos vivos frente a 5 por mil en los establecimientos de menor capacidad resolutiva). Debido a estas diferencias, se estimó dos modelos separados. El primero correspondió a establecimientos de menor capacidad resolutiva (policlínicos y hospitales de nivel I y II), que incluyó 32 establecimientos: policlínicos El Porvenir, La Esperanza, Moche, Castilla, Negreiros, Huaral, Pacasmayo, y hospitales Albrecht, Alcántara, Aurelio Díaz Ufano, Florencio de Mora, Marino Molina, Paita, Sullana, Tarma, Uldarico Rocca, Voto Bernales, La Merced, Alberto Hurtado, Angamos, Cajamarca, Cañete, Chocope, G. Lanatta, Huamanga, Huánuco, Ilo, Pasco, Pucallpa, Reátegui, Talara y Tarapoto. El tamaño de la muestra para este grupo fue de 44378 nacimientos. El segundo correspondió a hospitales de mayor capacidad resolutiva (nivel III, IV y nacionales), que agrupó a 17 hospitales: Cayetano Heredia, Chimbote, Daniel
Alcides Carrión, Félix Torrealva, Grau, Iquitos, Juliaca, Puno, Yanahuara, Huancayo, Sabogal, Víctor Lazarte, Almanzor Aguinaga, Almenara, Cusco, Nacional del Sur (Carlos A. Seguín) y Rebagliati. El tamaño de la muestra en este grupo fue de 64435 nacimientos.

La significancia estadística de cada factor de riesgo fue evaluada con pruebas $t$, y se estimó valores de probabilidad ( $p$ values) al $1 \%, 5 \%$ y $10 \%$ de significancia. Se descartó problemas de multicolinearidad usando factores de inflación de varianza (variance - VIF) y se consideró errores estándar robustos para corregir por potenciales problemas de heteroscedasticidad. La estimación del modelo Logit multivariado se hizo usando el programa estadístico Stata v.9.2.

\section{RESULTADOS}

EsSalud ha seguido la tendencia nacional decreciente de mortalidad perinatal. Entre el año 2000 y el 2006, la tasa de mortalidad perinatal ha caído en cerca de $40 \%$, lo cual implica una tasa de reducción mayor a la observada a nivel nacional, que ha sido cercana al 30\%. La figura 1 muestra la mortalidad perinatal de EsSalud en sus dos componentes, mortalidad fetal y mortalidad neonatal precoz, los cuales han disminuido $42 \%$ y $33 \%$, respectivamente, entre el año 2000 y 2006. Cabe mencionar que la tasa de mortalidad neonatal descendió de 8,7 a 5,8 y 6,9 por mil nacidos vivos, en el mismo periodo.

La reducción de la mortalidad perinatal en EsSalud se ha logrado principalmente gracias a un mayor control de la prematuridad, infección y asfixia. La figura 2 muestra la tasa de mortalidad neonatal por principales causas. Resalta la presencia de anomalías congénitas asociada más a características epidemiológicas de la población que a factores institucionales, así como la prematuridad, relacionada tanto con la capacidad resolutiva institucional como al aumento de partos prematuros.

Los resultados de las estimaciones logísticas son presentados en la tabla, en la cual se señala los odds ratios (OR) e intervalos de confianza para cada nivel de establecimiento analizado. Dentro de los riesgos de mortalidad perinatal asociados a la madre, la edad así como las enferme- 


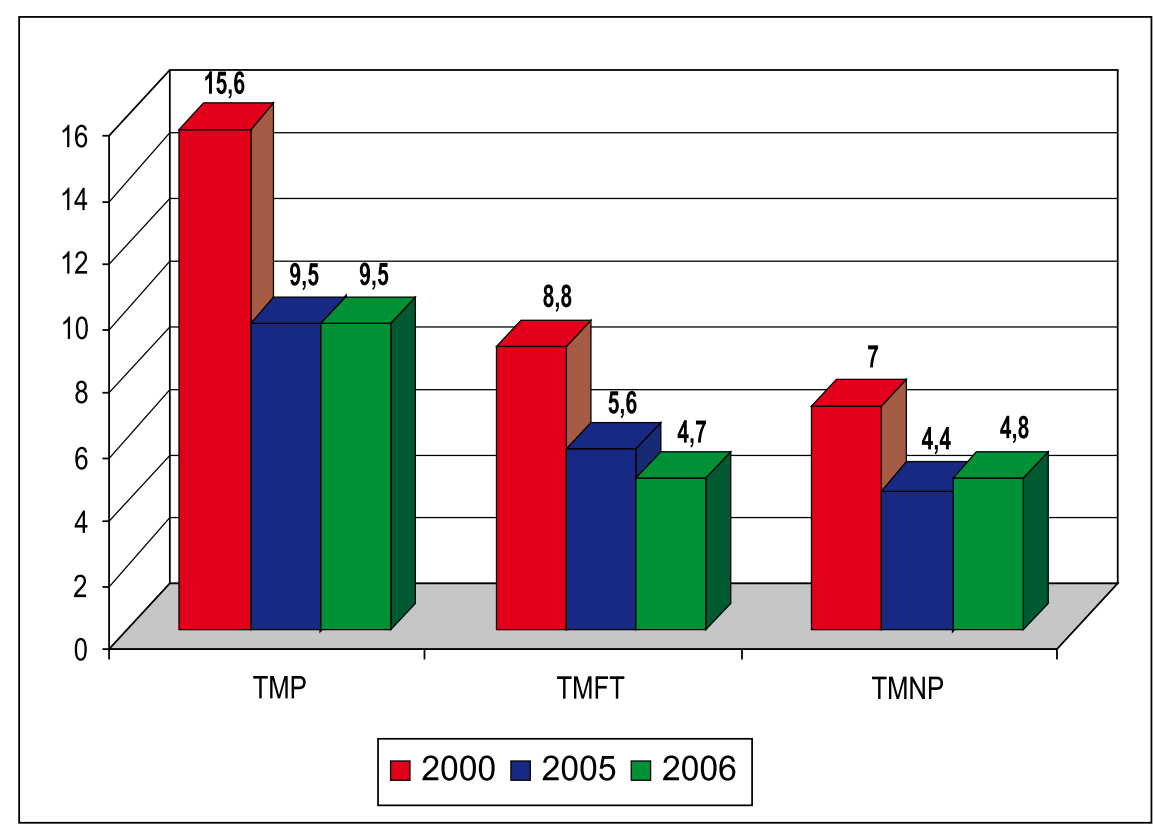

Figura 1. Tasa de mortalidad perinatal, fetal tardía y neonatal precoz.

dades durante el embarazo no resultaron estadísticamente significativas. El número de partos significó un riesgo de mortalidad perinatal, OR de 1,12 y 1,18 , en establecimientos de segundo y tercer nivel de atención, respectivamente. El impacto de entidades obstétricas varía de acuerdo al nivel de atención. En establecimientos de segundo nivel, el sangrado genital presentó un OR igual a 4,23 y otras entidades un $\mathrm{OR}$ de 3,81, constituyéndose también

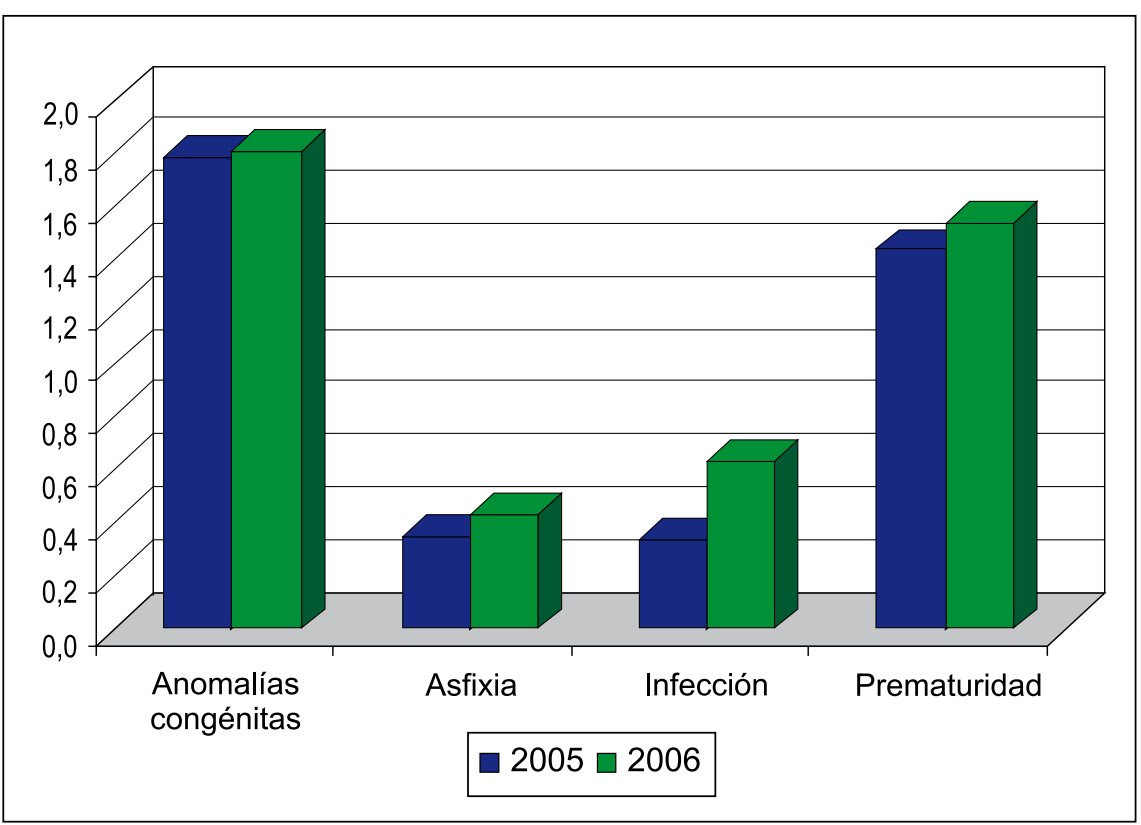

Figura 2. Tasa de mortalidad neonatal precoz por causa. contrario a lo esperado, se obtuvo $\mathrm{OR}$ de 0,65 y 0,46 , respectivamente.

En el caso de riesgos de mortalidad perinatal asociados al recién nacido, todos los factores incluidos resultaron significativos y con la dirección esperada. Sin embargo, el impacto de los factores de riesgo sobre la mortalidad perinatal fue mucho mayor en hospitales de segundo nivel que en los de tercer nivel. Por ejemplo, el OR de edad gestacional menor a 32 semanas fue 15,68 en el caso de establecimientos de segundo nivel, pero 4,20 en los de tercer nivel. El OR de sobrepeso (peso mayor a 4000 gramos) fue 4,17 y 3,87 en establecimientos de segundo y tercer nivel, respectivamente. Del mismo modo, la anomalía congénita tuvo un OR de 30,99 en hospitales de segundo nivel, comparado con 15,26 en los de tercer nivel. El sexo del recién nacido también incide en la probabilidad de muerte perinatal. El OR de sexo fue 0,72 y 0,87 en establecimientos de segundo y tercer nivel, respectivamente, aunque en este último la significancia estadística fue solo de $10 \%$.

\section{DISCUSIÓN}

La mortalidad perinatal en EsSalud ha seguido la tendencia decreciente observada a nivel nacional. Sin embargo, según lo muestran las cifras mostradas por la Endes, la reducción de la mortalidad en EsSalud ha sido mucho más rápida, alcanzando una tasa de 10 fallecidos por cada 1000 nacidos vivos. Esto ubica a la institución dentro de los estándares de países como Chile o Colombia, largamente reconocidos por su calidad perinatal dentro de América Latina. Sin embargo, los resultados de este estudio muestran que aun hay más espacio para reducir esta tasa, sobre todo en establecimientos con menor capacidad resolutiva de atención materno-perinatal.

El estudio encuentra coincidencias con la literatura nacional e internacional sobre factores de riesgo asociados a la mortalidad perinatal ${ }^{(8,10,11,13,14)}$. En primer lugar, las anomalías congénitas constituyen el principal factor de riesgo asociado a la mortalidad perinatal. En los establecimientos con menor capacidad resolutiva (policlínicos, hospitales I y II), un feto con anomalías congénitas tiene 31 veces más posibilidades de morir 
Tabla. Factores de riesgo de mortalidad perinatal utilizados.

\begin{tabular}{|c|c|c|c|c|}
\hline \multirow[t]{2}{*}{ Variable } & \multicolumn{2}{|c|}{ Segundo nivel de atención * } & \multicolumn{2}{|c|}{ Tercer nivel de atención ${ }^{\dagger}$} \\
\hline & Odds ratio & IC 95\% & Odds ratio & IC al $95 \%$ \\
\hline \multicolumn{5}{|l|}{ Características de la madre } \\
\hline \multicolumn{5}{|l|}{ Edad en años } \\
\hline 19 años o menor & 1,37 & {$[0,39,4,80]$} & 1,38 & {$[0,81,2,35]$} \\
\hline 35 años o mayor & 1,26 & {$[0,68,2,34]$} & 1,02 & {$[0,75,1,37]$} \\
\hline Número de partos & $1,12 \ddagger$ & {$[0,98,1,29]$} & $1,18^{\S}$ & {$[1,11,1,26]$} \\
\hline Número de abortos & 1,11 & {$[0,87,1,43]$} & 0,98 & {$[0,87,1,11]$} \\
\hline Enfermedades durante el embarazo & 1,08 & {$[0,71,1,65]$} & 0,88 & {$[0,71,1,08]$} \\
\hline \multicolumn{5}{|l|}{ Entidades obstétricas } \\
\hline Anemia & 1,45 & {$[0,61,3,43]$} & $1,48 \ddagger$ & {$[0,95,2,32]$} \\
\hline Preeclampsia & 1,69 & {$[0,86,3,33]$} & $0,65 \S$ & {$[0,48,0,87]$} \\
\hline Sangrado genital & $4,23 \S$ & {$[1,50,11,93]$} & $3,81^{\S}$ & {$[2,58,5,65]$} \\
\hline Rotura prematura membranas & 1,05 & {$[0,52,2,15]$} & $0,46 \S$ & {$[0,33,0,64]$} \\
\hline Otras entidades & $4,53 \S$ & {$[2,96,6,95]$} & $1,76 \S$ & {$[1,42,2,18]$} \\
\hline \multicolumn{5}{|l|}{ Características del recién nacido } \\
\hline \multicolumn{5}{|l|}{ Edad gestacional } \\
\hline 31 semanas o menos & $15,68^{\S}$ & {$[6,88,35,72]$} & $4,20 \S$ & {$[2,93,6,04]$} \\
\hline Entre 32 y 36 semanas & $4,95 \S$ & {$[2,95,8,31]$} & $2,84 \S$ & {$[2,20,3,68]$} \\
\hline Más de 42 semanas & $6,91^{\S}$ & {$[2,44,19,61]$} & 1,82 & {$[0,67,4,97]$} \\
\hline \multicolumn{5}{|l|}{ Peso del recién nacido } \\
\hline Peso por $100 \mathrm{~g}$ & $0,86^{\S}$ & {$[0,82,0,91]$} & $0,88^{\S}$ & {$[0,86,0,91]$} \\
\hline Peso menor a $1000 \mathrm{~g}$ & 2,35 & {$[0,49,11,17]$} & $4,26^{\S}$ & {$[2,01,9,03]$} \\
\hline Peso entre 1000 y $1500 \mathrm{~g}$ & 1,58 & {$[0,38,6,5]$} & 1,67 & {$[0,90,3,08]$} \\
\hline Peso entre 1500 y $2500 \mathrm{~g}$ & 0,93 & {$[0,46,1,91]$} & $1,49 \|$ & {$[1,02,2,18]$} \\
\hline Peso mayor a $4000 \mathrm{~g}$ & $4,17 \S$ & {$[1,46,11,87]$} & $3,87^{\S}$ & {$[2,13,7,02]$} \\
\hline Sexo & $0,72 \ddagger$ & {$[0,51,1,02]$} & 0,87 & {$[0,73,1,03]$} \\
\hline Anomalía congénita & $30,99 \S$ & {$[14,0,68,4]$} & $15,26^{\S}$ & {$[11,8,19,8]$} \\
\hline
\end{tabular}

"Policlínicos y hospitales de nivel I y II. El total de observaciones en este grupo fue de 44378 nacimientos.

${ }^{\dagger}$ Hospitales de nivel III, IV y nacionales. El total de observaciones en este grupo fue de 64435 nacimientos.

${ }^{\ddagger}$ significativo al $10 \% \quad$ § significativo al $1 \%$ significativo al $5 \%$

durante la etapa perinatal que un feto sin anomalías, mientras que en establecimientos de mayor capacidad resolutiva (hospitales III, IV y nacionales) esta posibilidad se reduce a 15,3 veces. Las muertes por anomalía congénita han aumentado en los últimos años en EsSalud, sugiriendo la existencia de deficiencias epidemiológicas en la población. Dada su fuerte incidencia sobre mortalidad y su mayor relevancia, es importante extender los estudios para entender las causas y definir políticas efectivas de salud pública orientadas a su reducción.

En segundo lugar, la edad gestacional seguido por el peso son también factores de riesgo significativos asociados a mortalidad perinatal. Existen también marcadas diferencias en su incidencia entre hospitales de menor y mayor capacidad resolutiva. Por ejemplo, un embarazo prematuro menor a 32 semanas atendido en un establecimiento de menor capacidad resolutiva tiene 15,7 veces más posibilidades de terminar en pérdida que un embarazo normal (entre 37 y 42 semanas). Sin embargo, si la atención fuese en un establecimiento de mayor capacidad resolutiva, la posibilidad de pérdida sería de solo 4,2 veces. Una diferencia similar se observa en el caso de prematuros con 32 a 36 semanas, o en el de posmaduros con 42 o más semanas, que solo es un factor de riesgo estadísticamente significativo en establecimientos de menor capacidad resolutiva. Esto se explica también por el hecho de que las guías médicas en hospitales de mayor capacidad tienden a terminar la gestación antes de las 42 semanas, como medida para reducir la mortalidad.

Coincidente con otros estudios ${ }^{(10,13)}$, el sexo del recién nacido incide sobre la capacidad de sobrevivencia. En hospitales de menor capacidad resolutiva, un recién nacido de sexo masculino tiene $28 \%$ menos posibilidades de sobrevivir a la etapa perinatal que un bebe de sexo femenino. La posibilidad se reduce a la mitad cuando la atención es en hospitales de mayor capacidad resolutiva, aunque en este caso no resulta estadísticamente significativa.

Los factores de riesgo asociados a la madre tienen menor asociación con la mortalidad perinatal. El número de partos anteriores resulta estadísticamente significativo y afecta positivamente la probabilidad de muerte, mas no el número de abortos. Las enfermedades concomitantes del embarazo no resultaron relevantes, pero sí algunas entidades obstétricas. En particular, el sangrado genital en la segunda mitad del embarazo y otras entidades -como la anemia, preeclampsia y rotura prematura de membranas- incrementan la probabilidad de muerte perinatal. Nuevamente, la incidencia de estos factores de riesgo es mayor en hospitales de menor capacidad resolutiva. La anemia aumenta también el riesgo de muerte, pero resultó no significativa en hospitales de menor capacidad y significativa solo a $10 \%$ en hospitales de mayor capacidad resolutiva. Es importante resaltar dos resultados no esperados. La preeclampsia y la rotura prematura de membranas reducen la probabilidad de muerte en hospitales de mayor capacidad resolutiva. En hospitales de menor capacidad, la incidencia es la esperada, pero no es estadísticamente significativa.

Tanto los resultados no esperados en hospitales de mayor capacidad resolutiva como el menor impacto de los factores de riesgo comparado con los establecimientos de menor capacidad resolutiva están asociados a un mismo fenómeno. En la red hospitalaria de EsSalud, los casos de mayor riesgo son y deben ser transferidos a los establecimientos con mayor capacidad resolutiva. Son aquellos los que ofrecen las condiciones más adecuadas para garantizar la sobrevivencia del binomio madre-niño. Adicionalmente, las guías médicas de atención materno-perinatal destinan mayores recursos a aquellos casos identificados como de mayor riesgo durante el embarazo. Por esta razón, si bien las enfermedades durante el embarazo debieran incidir sobre la mortalidad perinatal, un tratamiento oportuno y focalizado de las mismas hará que estos casos de riesgo no terminen en muerte y, por consiguiente, no resulten significativos en las estimaciones. Otro ejemplo es 
la preeclampsia, una de las principales causas de muerte materna. Las guías de salud se han concentrado en este factor de riesgo alto, de manera que hoy en día una mujer con preeclampsia será atendida con mayores recursos y cuidados que pueden, finalmente, hacer que su probabilidad de muerte y la de su hijo sean menores que las de una madre sin preeclampsia con acceso a recurso promedio.

Es decir, el impacto estimado combina tanto el factor de riesgo clínico como el esfuerzo institucional para destinar más recursos a los casos identificados como de mayor riesgo (por ejemplo, preeclampsia y rotura de membranas). Este esfuerzo institucional es superior en establecimientos de mayor capacidad resolutiva, donde refieren los pacientes más riesgosos.

Las implicancias de estos resultados son importantes en términos de políticas. En primer lugar, se resalta la eficacia de EsSalud en reducir la mortalidad perinatal a través de una mayor prioritización de recursos en factores de riesgo materno claves, lo cual explica la reducción en la mortalidad materno-perinatal en la institución. Políticas similares debieran ser compartidas con la red hospitalaria del Ministerio de Salud. Es interesante ver que tanto Minsa como EsSalud tienen sus indicadores perinatales en descenso, aunque aún existe una brecha bastante importante entre ellos, tanto en las tasas de mortalidad como en las causas de muerte. Esta brecha está en relación a diferencias en los perfiles epidemiológicos de sus pacientes, así como en el acceso a recursos. Sin embargo, la eficiencia en el uso de estos recursos, priorizando factores de riesgo más alto a través de indicadores de alerta temprana, podría reducir la brecha e incluso los niveles de mortalidad a nivel nacional.

Un segundo resultado importante de este estudio es que se ha encontrado espacios para reducir aún más la mortalidad perinatal en EsSalud. Esto se puede lograr atacando varios frentes: i) enfocando recursos en las dos entidades obstétricas con mayor incidencia sobre mortalidad perinatal: sangrado genital después de la semana 24 del embarazo y otras entidades, que requiere un mayor detalle de inspección; ii) prioritizando más la asignación de recursos en factores de riesgo alto en los policlínicos y hospitales I y II con capacidad de atención materno- perinatal; iii) mejorando el proceso de referencias de pacientes de hospitales con menor capacidad resolutiva a hospitales de mayor capacidad; iv) monitoreando mejor los factores de riesgo del recién nacido (en particular edad gestacional, anomalía congénita, peso y sexo), por medio de sistemas de alerta temprana de riesgos perinatales basados en una mayor explotación de la información del SVP.

Finalmente, este estudio ha mostrado la utilidad y beneficios del SVP de EsSalud, el cual permite no solo identificar factores de riesgo perinatal a nivel nacional, sino también elaborar recomendaciones de políticas más efectivas para reducir la mortalidad. Para conseguir ello, un sistema informático debe ser ágil y amigable, con variables basadas principalmente en principales factores de riesgo y entidades prevalentes, de modo que su digitación demore de 2 a 5 minutos. Debe brindar información que permita obtener indicadores intermedios y en tiempo real, de manera que facilite una efectiva vigilancia de la salud materna y neonatal a nivel de servicios, hospitales, redes y a nivel nacional. En ese sentido, son importantes los esfuerzos de otras instituciones como las Fuerzas Armadas y el Minsa, para desarrollar e implementar sistemas de vigilancia perinatal, pero es también necesario unir esfuerzos para que estos sistemas sean compatibles entre sí. Hoy en día son reconocidas las ventajas de integrar bases de datos clínicas entre instituciones ${ }^{(15,16)}$, tanto para mejorar la eficiencia en el uso de recursos, aumentar la calidad de la salud y sobre todo para unir esfuerzos interinstitucionales en la lucha por disminuir la mortalidad materna y perinatal en país.

\section{AGRADECIMIENTOS}

Los autores agradecen a EsSalud por el acceso a la información del SVP, principalmente al Ing. Zoilo Silva. El estudio contó con el soporte del Consorcio de Investigación Económica y Social (CIES), a través del programa ACDI-IDRC 2008.

\section{REFERENCIAS BIBLIOGRÁFICAS}

1. UNICEF. The State of The World's Children 2009: Maternal and Newborn Health. United Nations Children's Fund; 2008.

2. Guzmán A. Para mejorar la salud reproductiva. En: Arroyo J, editor. La salud peruana en el siglo XXI. Retos y propuestas de política. Lima: Consorcio de Investigación Económica y Social; 2002. p. 185-238.

3. Rebaza H. Para una política hospitalaria. En: Arroyo J, editor. La salud peruana en el siglo XXI. Retos y propuestas de política. Lima: Consorcio de Investigación Económica y Social; 2002. p. 239-302.

4. Åmman E, Zupan J. Neonatal and perinatal mortality: country, regional and global estimates 2004. World Health Organization; 2007.

5. Santiago M. Barboza E, Campos D. Avaliação da qualidade da informação do Sistema de Informação Perinatal (SIP-CLAP /OPAS) para monitoramento da assistência perinatal hospitalar, Belo Horizonte, 2004. Revista Brasileira de Saúde Materno Infantil. 2009;9(3):275-84.

6. Espíritu N, Sacieta L, Pantoja L. Discrepancias en el registro de la mortalidad perinatal en Lima y Callao, según fuente de Información. Rev Peru Med Exp Salud Publica. 2007;24(4):363-9.

7. Simina F. Perinatal information system (SIP): a clinical database in Latin America and the Caribbean. The Lancet. 1999;354(9172):75.

8. Ticona RM, Huanco A. Mortalidad perinatal hospitalaria en el Perú: factores de riesgo. Revista Chilena de Obstetricia y Ginecología. 2005;70(5):313-7.

9. Jewell T, Triunfo P. The impact of prenatal care on birthweight: The case of Uruguay. Health Economics. 2006;15(11):1245-50.

10. Bacak SJ, Baptiste-Roberts K, Amon E, Ireland B, Leet T. Risk factors for neonatal mortality among extremely-low-birth-weight infants. Am J Obstet Gynecol. 2005;192(3):862-7.

11. Forssas E, Gissler M, Sihvonen M, Hemminki E. Maternal predictors of perinatal mortality: the role of birthweight. Int J Epidemiol. 1999;28(3):475-8.

12. Allison P. Missing data. New York: Sage; 2001.

13. Silver RM, Varner MW, Reddy U, Goldenberg R, Pinar H, Conway D. Work-up of stillbirth: a review of the evidence. Am J Obstet Gynecol. 2007;196(5): 433-44.

14. Cnattingius S, Stephanson 0 . The epidemiology of stillbirth. Sem Perinatol. 2002;26(1):25-30.

15. Pryor DB, Califf RM, Harrell FE Jr, Hlatky MA, Lee KL, Mark DB. Clinical data bases. Accomplishments and unrealized potencial. Med Care. 1985;23(5):62347.

16. Black N. High-quality clinical databases: breaking down barriers. The Lancet. 1999;353(9160):1205-6.

Manuscrito recibido el 16 de noviembre de 2009 y aceptado para publicación el 1 de diciembre de 2009.

Correspondencia:

Dra. Gloria Riesco de la Vega

Programa Nacional Materno - Perinatal

EsSalud-Sede Central

Av. San Felipe 915-203

Lima 11, Perú

Correo-e: gloriariesco@yahoo.es 